\title{
Elevated Plasma Levels but Decreased Platelet-Associated Expression of LIGHT in Patients with Acute Atherothrombotic Stroke
}

\author{
Guangzhi Liu1* ${ }^{*}$ Yang He1, Tingting Yang1, Hong Jiang1, Yajuan Xiang1, Qi Zhang2, \\ Xiangjun $\mathrm{He}^{2}$, Peter Hjelmstrom ${ }^{3}$, Xuguang Gao' \\ ${ }^{1}$ Department of Neurology, Peking University People's Hospital, Beijing, China \\ ${ }^{2}$ Key Laboratory Centre, Peking University People's Hospital, Beijing, China \\ ${ }^{3}$ Department of Clinical Science, Intervention and Technology, Karolinska Institute, Stockholm, Sweden \\ Email: "guangzhi@public.bta.net.cn
}

Received 28 June 2014; revised 28 July 2014; accepted 11 August 2014

Copyright (C) 2014 by authors and Scientific Research Publishing Inc.

This work is licensed under the Creative Commons Attribution International License (CC BY).

http://creativecommons.org/licenses/by/4.0/

(c) (i) Open Access

\section{Abstract}

Background: As a member of the tumor necrosis factor superfamily (TNFSF), LIGHT (TNFSF14) is expressed by a variety of immune cells and exists in membrane-bound and soluble forms. Recently, LIGHT was found to be associated with platelets and released upon activation. Activation of endothelia cells by recombinant LIGHT protein results in pro-inflammatory and pro-thrombotic changes. Several studies have reported increased plasma levels of LIGHT in patients with stroke and cardiovascular diseases. However, the form-associated roles of LIGHT in ischemic atherosclerotic stroke remain unclear. Materials and Methods: In this study, the platelet LIGHT expression and soluble LIGHT protein were analyzed by flow cytometry and enzyme-linked immunosorbent assay (ELISA) in peripheral blood of patients with acute ischemic atherosclerotic stroke, asymptomatic carotid stenosis (ACS) and normal controls. RESULTS: During the initial $24 \mathrm{~h}$ after onset, the stroke patients had decreased LIGHT expression on their platelets $(5.9 \% \pm 4.9 \%)$ and increased plasma LIGHT levels $(36.1 \pm 21.0 \mathrm{pg} / \mathrm{ml})$ as compared with normal controls $(9.5 \% \pm 3.0 \%$, $p<0.05 ; 20.4 \pm 13.4 \mathrm{pg} / \mathrm{ml}, p<0.05)$. Moreover, the platelet LIGHT expression correlated with total plaque area in the stroke patients $(r=0.4572, p=0.0247)$. Conclusions: The dysregulated LIGHT expression reflects a persistent chronic inflammatory response that may have been induced during early stages of ischemic atherosclerotic stroke. Our results strongly suggest distinctive roles of form-associated LIGHT in the disease pathogenesis: platelet-associated LIGHT may contribute to formation and development of carotid atherosclerotic plaque, probably involving

${ }^{*}$ Corresponding author.

How to cite this paper: Liu, G.Z., He, Y., Yang, T.T., Jiang, H., Xiang, Y.J., Zhang, Q., He, X.J., Hjelmstrom, P. and Gao, X.G. (2014) Elevated Plasma Levels but Decreased Platelet-Associated Expression of LIGHT in Patients with Acute Atherothrombotic Stroke. World Journal of Neuroscience, 4, 385-393. http://dx.doi.org/10.4236/wjns.2014.44042 
plaque destabilization, while soluble LIGHT may predominantly functions as a pro-inflammatory cytokine in the inflammatory process.

\title{
Keywords
}

\author{
LIGHT, Ischemic Atherosclerotic Stroke, Atherogenesis, Peripheral Blood
}

\section{Introduction}

Inflammation has received increasing attention in recent years as a cause of atherosclerosis [1] [2]. So far, various studies have provided strong evidences that atherosclerosis involves immune cells, such as T cells, monocytes or macrophages in the vasculature, systemically elevated proinflammatory cytokines, chemokines, adhesion molecules, and tissue factor (TF) [3]. In its advanced stages, atherosclerosis leads to the formation of an intravascular thrombus, which plays a critical role in the setting of acute arterial thrombosis, heart attack and stroke [4].

A variety of cytokines, including members of the tumor necrosis factor (TNF) superfamily (TNFSF), have been identified in atherosclerotic lesions and implicated in the pathogenesis of atherosclerosis [3] [5]. TNF- $\alpha$ and CD40 ligand (CD40L) have been found to have pivotal roles in atherogenesis by eliciting immune responses such as secretion of proinflammatory cytokines, activation of matrix metalloproteinases (MMP) and induction of TF expression [6]-[8]. In addition, another TNFSF member, LIGHT (TNFSF14), has recently been reported to demonstrate an enhanced expression in human atherosclerotic plaques and the ability to induce MMP activity in macrophages [9]-[11]. More interestingly, LIGHT was found to be associated with platelets and released as a soluble ligand upon platelet activation [10], and activation of human endothelial cells by the soluble LIGHT resulted in pro-inflammatory and pro-thrombotic changes [9] [12]. Of note, two separate studies revealed significantly increased plasma levels of LIGHT in patients with ST-elevation myocardial infarction (STEMI) and unstable angina as compared with normal controls [13] [14]. Moreover, we previously reported higher plasma levels of LIGHT in patients with acute ischemic atherosclerotic stroke than those of normal controls, and the plasma LIGHT levels of the stroke patients were correlated with blood platelet count [15]. Nevertheless, there is still a pressing need for further studies of the form-associated LIGHT expression as well as their possible roles in the atherogenetic process of ischemic stroke. Therefore, we have analyzed platelet LIGHT expression and soluble LIGHT protein in peripheral blood of patients with this disease to address these issues.

\section{Material and Methods}

\subsection{Patients and Controls}

Twenty-four patients with acute ischemic atherosclerotic stroke (20 men and 4 women; mean age $68.3 \pm 13.7$ years) were enrolled in this study. These patients had large-artery atherosclerosis (LAA) subtype, according to the Acute Stroke Treatment (TOAST) classification [16]. All patients were evaluated within 24h of ischemic stroke onset and diagnosed on the basis of medical history, clinical examination and results of brain MRI and MRA scans. Carotid duplex sonography was performed in all patients, who were further subdivided into stable plaque ( $n=11,9$ men and 2 women) and unstable plaque groups ( $n=13,11$ men and 2 women) in terms of plaques echolucency using carotid ultrasound with integrated backscatter (IBS). Total plaque area (TPA) was measured as described previously [17] [18]. National Institute of Health Stroke Scale (NIHSS) was adopted to reflect the disease severity at the time of blood sampling. Infarct volume was obtained on axial diffusionweighted imaging (DWI) by summing the number of outlined voxels and multiplying by the slice thickness on each slice on which the lesion was visible. Patients with risk factors for cardioembolic stroke such as atrial fibrillation, vascular heart disease, acute myocardial infarction and endocarditis were excluded from this study. In addition, three of these patients with LAA stroke receiving treatment with aspirin (100 mg qd) were chosen for serial study.

As non-stroke disease controls, the atherosclerosis controls consisted of twenty-one patients (18 men and 3 women; mean age $72.4 \pm 8.7$ years) with asymptomatic carotid stenosis (ACS) (>50\%) on carotid duplex sono- 
graphy. All the patients had no evidence of ischemic stroke events on clinical history and brain MRI. Eighteen healthy subjects (13 men and 5 women; mean age $62.7 \pm 17.3$ years) were included as normal controls (NC) without any evidence of large vessel atherosclerosis, clinical history of vascular events or vascular risk factors.

According to their medical history and medical records, none of the participating individuals had (1) history of taking anti-platelets, calcium channel blockers and statins as well as other drugs known to interfere with platelet functions, including Cefazolin, Ampicillin, heparin,propranolol, verapamil, alcohol, phenylbutazone, Daunorubicin, hydroxyethyl starch, Dextranum, chlorqromazine, et al.; (2) autoimmune, hepatic, renal or cancerous disorders; or (3) an obvious infection. The following diagnostic tests were performed in both patients and normal controls: complete blood counts, blood chemistry, C reactive protein (CRP), electrocardiogram (EKG), posterior-anterior chest radiography, transthoraric cardiac echocardiography, transcranial Doppler ultrasonography and carotid duplex sonography. All participating subjects gave their informed consent before the start of the study.

\subsection{Sample Collection}

All the citrated and heparinized blood specimens were collected between 09:00 am and 12:00 am. For serial study, blood specimens of the patients with ischemic stroke were collected after $30 \mathrm{~d}$ of ischemic stroke onset. We chose $30 \mathrm{~d}$ as the collection time, because most patients get stably improved in the stage and usually take aspirin and statin without other drugs known to interfere with platelet functions, such as antibiotics, heparin, propranolol, Dextranum, et al. Platelet rich plasma (PRP) was obtained from the citrated blood specimens by centrifugation at $180 \mathrm{~g}$ for $10 \mathrm{~min}$. After the centrifugation from heparinized blood, plasma was stored at $-70^{\circ} \mathrm{C}$ in small aliquots and thawed just before further use.

\subsection{Flow Cytometry}

Platelets of the patients and control subjects were characterized for the expression of LIGHT by two-color direct immunofluorescence and flow cytometry using a FACScan (Becton Dickinson, CA, USA). PRP was washed with phosphate buffer saline (PBS, pH 7.2) and resuspended in PBS. The following monoclonal antibodies were added to $10 \mu \mathrm{l}$ platelet suspension: 1) fluorescence isothiocyanate (FITC)-labeled IgG1 (Becton Dickinson, CA, USA), allophycocyanin (APC)-labeled IgG2b (R\&D systems, Abingdon, UK); 2) FITC-labeled anti-CD61 (Becton Dickinson, CA, USA), APC-labeled anti-LIGHT (R\&D systems, Abingdon, UK). Following a 20 min incubation at $4^{\circ} \mathrm{C}$, the cells were washed with staining buffer and analyzed on the FACScan using the Cell Quest software.

\subsection{Enzyme-Linked Immunosorbent Assay (ELISA)}

Plasma LIGHT levels were measured by a commercial ELISA according to the supplier's directions (R\&D systems, Abingdon, UK) with a detection limit of $16.5 \mathrm{pg} / \mathrm{ml}$. Briefly, microtiter plates (Costar, NY, USA) were coated overnight with $100 \mu \mathrm{L} /$ well of the capture antibody at $4 \mu \mathrm{g} / \mathrm{ml}$. Non-specific binding was blocked by 300 $\mu \mathrm{L}$ of PBS containing $1 \%$ BSA, $5 \%$ sucrose and $0.05 \% \mathrm{NaN}_{3}$. Plasma or standards with a two-fold dilution series starting at $2.5 \mathrm{ng} / \mathrm{ml}$ were added with $100 \mu \mathrm{L}$ per well in duplicate. $100 \mu \mathrm{L}$ biotinylated detection antibody with a concentration of $50 \mathrm{ng} / \mathrm{ml}$ was added to each well, followed by stepwise addition of $100 \mu \mathrm{L}$ streptavidin HRP (R\&D Systems, UK) and $100 \mu \mathrm{L}$ of Substrate Solution (R\&D Systems, UK) to each well, respectively. 50 $\mu \mathrm{L}$ of Stop Solution $\left(1 \mathrm{M} \mathrm{H}_{2} \mathrm{SO}_{4}\right)$ was thereafter added to each well to stop the reaction. Finally, the evaluation was done using a Labsystems Multiscan microplate reader (Labsystems, Finland) set to $450 \mathrm{~nm}$. To calculate assay results, duplicate readings were averaged, the zero standard optical density subtracted and compared to a standard curve done with known concentrations of human LIGHT protein. All assays were performed simultaneously in a blinded fashion.

\subsection{Statistics}

The data are presented as mean \pm standard deviation of the mean value (plasma LIGHT levels, surface expression of LIGHT on platelets). Data with a normal distribution were analyzed with one-way analysis of variance (ANOVA) by Student-Newman-Keul's post-hoc test and Pearson's correlation test. Data with a non-normal distribution were analyzed with Kruskal-Wallis ANOVA and Spearman's correlation test. $p$ values of less than 0.05 
were considered to be statistically significant.

\section{Results}

\subsection{Clinical and Laboratory Data}

Patients' characteristics including risk factors and laboratory data taken on the first day of sampling are shown in Table 1. In addition, no significant differences in blood pressure or levels of blood glucose, total cholesterol, high-density lipoprotein (HDL), low-density lipoprotein (LDL), CRP were found between the stroke patients and either of the control groups (data not shown).

\subsection{Surface Expression of LIGHT on Platelets}

Within $24 \mathrm{~h}$ of onset, patients with ischemic stroke showed decreased expression of LIGHT (Figure 1) on CD61+ platelets $(5.9 \% \pm 4.9 \%)$ compared with NC $(9.5 \% \pm 3.0 \%, p<0.05)$ (Figure 2(a)), but no significant difference was found between the stroke and ACS patients $(7.5 \% \pm 5.7 \%)$. Furthermore, there was a significant difference in the platelets LIGHT expression between unstable plaque subgroup (8.0\% $\pm 5.7 \%)$ and stable plaque subgroup (3.5\% $\pm 2.0 \%$ ) (Figure 2(b)). Compared with NC and ACS group, the stable subgroup demonstrated a decrease in platelet LIGHT expression $(p<0.05$ ) (Figure 2(c)). Moreover, three stroke patients, who were treated with aspirin, demonstrated a trend towards a decrease of LIGHT expression on CD61+ platelets after 30 days of treatment (Figure 2(d)).

Table 1. Baseline characteristics of patients and normal controls.

\begin{tabular}{ccccc}
\hline & $\begin{array}{c}\text { Ischemic stroke } \\
(\mathrm{n}=24)\end{array}$ & $\begin{array}{c}\text { Asymptomatic carotid } \\
\text { stenosis }(\mathrm{n}=21)\end{array}$ & $\begin{array}{c}\text { Normal controls } \\
(\mathrm{n}=18)\end{array}$ & $p$ value \\
\hline Sex (male/female) & $20 / 4$ & $18 / 3$ & $13 / 5$ & - \\
Age (years) & $68.3 \pm 13.7$ & $72.4 \pm 8.7$ & $62.7 \pm 17.3$ & - \\
Hypertension (\%) & 54.2 & 61.9 & - & - \\
Diabetes mellitus (\%) & 41.7 & 47.6 & - & - \\
Hypercholesterolemia (\%) & 25.0 & 23.8 & 50.6 & - \\
Smoking $(\%)$ & 45.8 & 66.7 & 22.2 & - \\
Peripheral artery disease $(\%)$ & 25 & 23.8 & $0.5 \pm 0.3$ & 0.262 \\
Monocyte $\left(\times 10^{3} / \mu 1\right)$ & $0.5 \pm 0.1$ & $0.4 \pm 0.2$ & $197.2 \pm 64.2$ & 0.688 \\
Platelet count $\left(\times 10^{3} / \mu 1\right)$ & $196.8 \pm 55.0$ & $183.8 \pm 50.4$ & - & 0.351 \\
Total plaque area $\left(\mathrm{mm}^{3}\right)$ & $78.23 \pm 139.1$ & $121.5 \pm 104.1$ & - \\
\hline
\end{tabular}

Data are mean \pm SD.

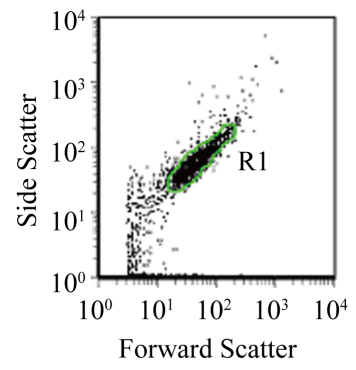

(a)

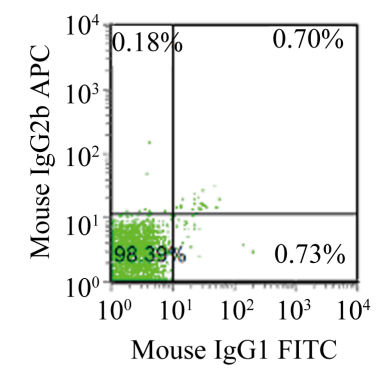

(b)

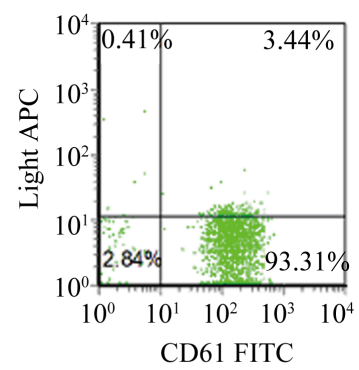

(c)

Figure 1. Region 1 (R1) is selected to set platelets gate for LIGHT analysis. Control staining with isotype control antibodies was used as control to define the gate. 


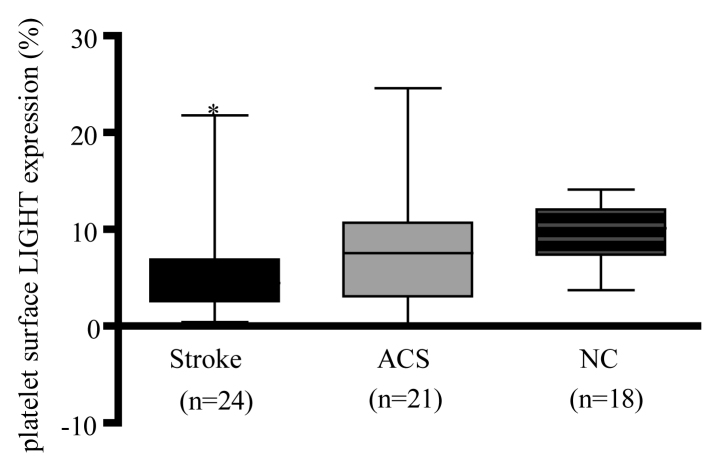

(a)

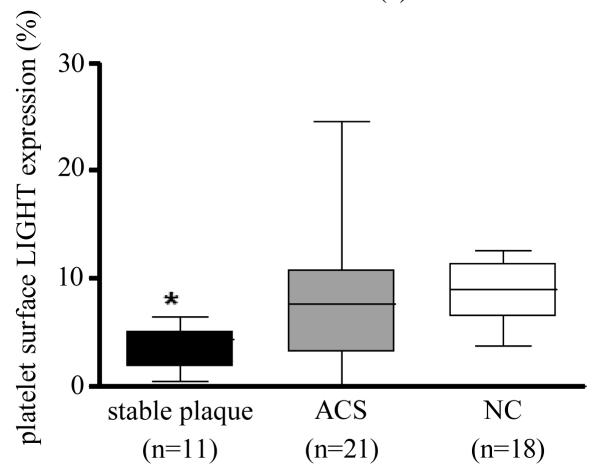

(c)

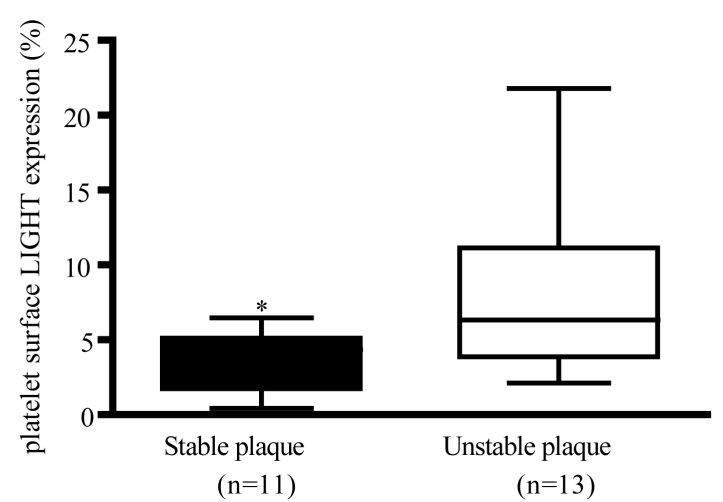

(b)

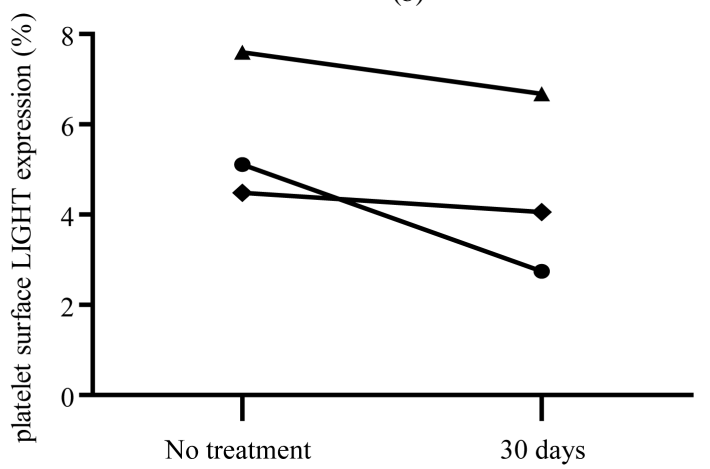

(d)

Figure 2. (a) Comparison of surface expression of LIGHT on platelets between patients with acute ischemic atherosclerotic stroke, asymptomatic carotid stenosis (ACS) and normal controls (NC) using a two-color flow cytometry. ${ }^{*} p<0.05$ compared with NC. (b) Comparison of surface expression of LIGHT on platelets between unstable plaque and stable plaque subgroups of the stroke patients using a two-color flow cytometry. ${ }^{*} p<0.05$ compared with unstable plaque subgroup. (c) Comparison of surface expression of LIGHT on platelets between stable plaque subgroup of the stroke patients and patients with asymptomatic carotid stenosis (ACS), normal controls (NC) using a twocolor flow cytometry. ${ }^{*} p<0.05$ compared with ACS and NC, respectively. (d) Changes of surface expression of LIGHT on platelets in three stroke patients before treatment and after $30 \mathrm{~d}$ of treatment with aspirin. $30 \mathrm{~d}=30$ days.

\subsection{Plasma LIGHT Levels}

During the initial $24 \mathrm{~h}$ after onset, the plasma LIGHT levels were significantly elevated in patients with ischemic stroke (36.1 $\pm 21.0 \mathrm{pg} / \mathrm{ml}$ ) compared with normal controls $(20.4 \pm 13.4 \mathrm{pg} / \mathrm{ml}, p<0.05)$ (Figure 3), but no significant difference was found between the stroke and ACS patients $(25.8 \pm 18.3 \mathrm{pg} / \mathrm{ml})$. Moreover, there was no remarkable difference in the plasma LIGHT levels between unstable plaque subgroup and stable plaque subgroup. Two of three stroke patients showed a decreasing trend after 30 days of ischemic events, whereas blood platelets and monocytes exhibited slight changes (Figure 4).

\subsection{Correlation Analysis}

The age, complete blood counts (platelet and monocyte), and surface expression of LIGHT on CD61+ platelets were not significantly correlated with plasma levels of LIGHT in either the patients with ischemic atherosclerotic stroke or control groups, except for a remarkable correlation with platelet count in the stroke patients $(\mathrm{n}=$ $24, r=0.5756, p=0.0033$ ). In addition, there was a significant correlation between the surface expression of LIGHT on platelets and TPA in the stroke patients $(\mathrm{n}=24, \mathrm{r}=0.4572, p=0.0247)$. Neither surface expression of LIGHT on platelets nor the plasma LIGHT levels correlated with NIHSS or infarct volume by brain MRI in the stroke patients (data not shown).

\section{Discussion}

In our study, patients with ischemic atherosclerotic stroke showed decreased surface expression of LIGHT on 


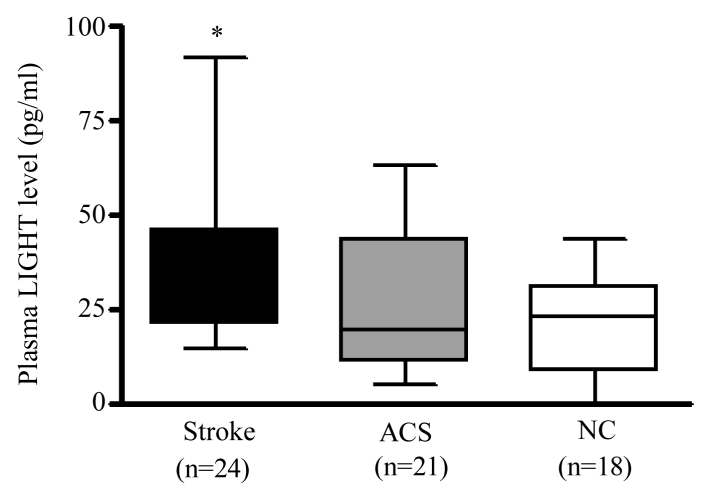

Figure 3. Comparison of plasma LIGHT levels between patients with acute ischemic atherosclerotic stroke, asymptomatic carotid stenosis (ACS) and normal controls (NC) using an enzyme-linked immunosorbent assay (ELISA). ${ }^{*} p<0.05$ compared with NC.

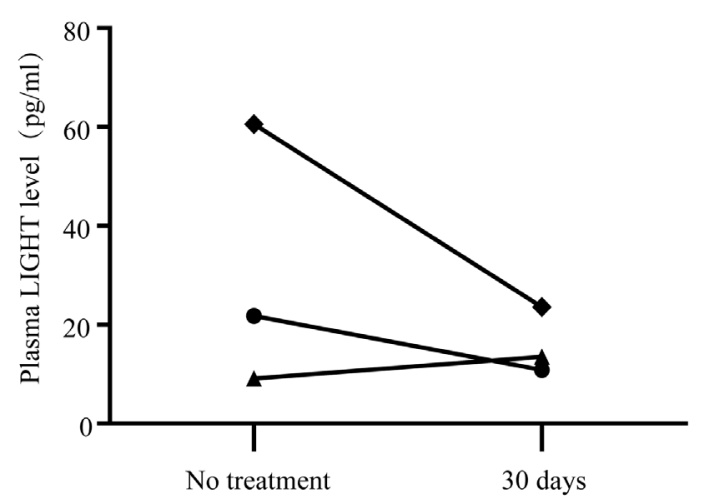

(a)

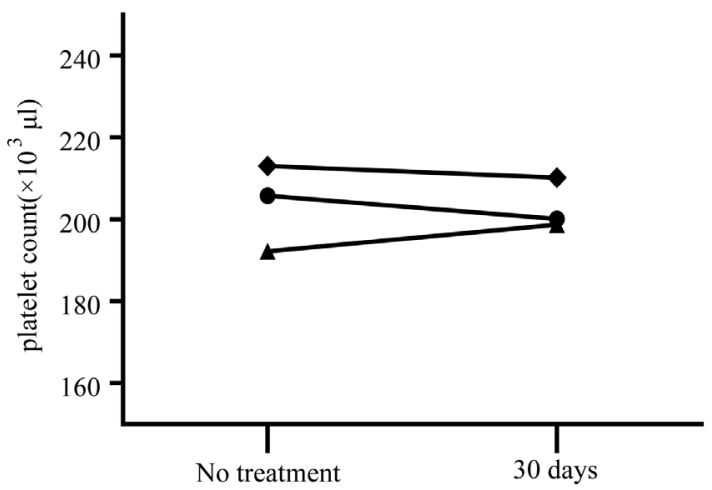

(b)

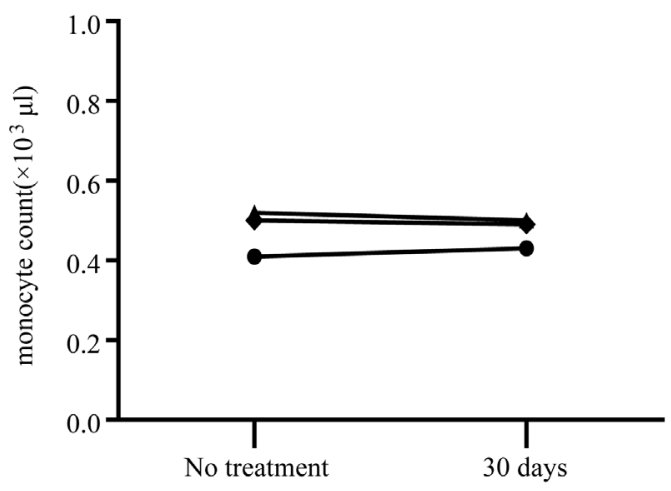

(c)

Figure 4. Changes of (a) plasma LIGHT levels, (b) blood platelet count and (c) monocyte count in three patients with acute ischemic atherosclerotic stroke before treatment and after $30 \mathrm{~d}$ of treatment with aspirin. $30 \mathrm{~d}=30$ days.

CD61+ platelets during the initial $24 \mathrm{~h}$ after onset. This indicates that a dysregulation of platelet-membrane LIGHT occurs in early stage of the disease. In consistent with our previous findings [15], higher plasma levels of LIGHT were also found in the stroke patients than those of NC and correlated with platelet count. Taking into consideration all these findings, this indicates that there is an aberrant platelet-related LIGHT expression existing in early stage of ischemic stroke.

LIGHT is produced primarily by activated T cells, monocytes, immature dendritic cells (DC) [19]-[21] and platelets [10] [14]. It exists in membrane-bound and soluble forms. The release of soluble LIGHT from activated lymphocytes was found to be dependent on MMP activity, but from activated platelets in a gradual and long- 
lasting manner involving a GP IIb/IIIa-dependent mechanism in addition to other factors such as MMP and actin polymerization [10] [22]. In our initial study, a significant correlation was found between the plasma LIGHT levels and blood platelet count in the stroke patients, suggesting that circulating platelets may be a major source of the soluble LIGHT protein [15]. Unexpectedly, we did not find a remarkable correlation between soluble LIGHT protein levels and surface expression of LIGHT on platelets. Together with similar findings reported in other TNFSF members such as platelet-derived CD40L [23], this study indicates that the soluble form of the molecule is not merely produced by shedding of the membrane-bound protein on the circulating platelets, but perhaps due to production from distinct, differentially spliced mRNA, or proteolytic cleavage from the cell surface. Intriguingly, a very recent study by Vanessa, et al. reported that high plasma LIGHT levels were associated with increased platelet activation (as shown by platelet P-selectin expression), rather than platelet counts [24]. Nevertheless, further studies on a larger scale are needed with non-treated stroke patients to confirm these findings.

So far, a variety of studies have proposed that the LIGHT protein might exert distinct effects on atherosclerosis in terms of the existing forms. Platelet-associated LIGHT may be involved in adhesion of platelets to endothelium, while soluble LIGHT may induce a pro-inflammatory state in vascular endothelial cells via a mechanism that contributes to atherogenesis formation and plaque destabilization as well as an inflammatory process involving leukocyte infiltration into the vessel wall [10] [14]. Our results favor the former notion, since there was a significant correlation between the platelet LIGHT expression levels and TPA of carotid artery in the stroke patients. Moreover, in the stroke patients relatively higher levels of platelet LIGHT expression were found in unstable plaque subgroup than those of stable subgroup. These indicate that platelet-associated LIGHT is closely related to formation and development of carotid atherosclerotic plaques, probably involving plaque destabilization. In line with our results, two distinct immunohistochemical studies revealed strong LIGHT expression on platelets, monocytes/macrophages of intracoronary thrombus and carotid atherosclerotic plaques, respectively [9] [10]. Together with the similar changes of platelet LIGHT expression in ACS patients, these findings strongly suggest that the decrease of platelet LIGHT expression in the acute stroke may occur, mainly due to adhesion of more circulating LIGHT-expressing platelets to vascular endothelium, deposition on the vessel wall with subsequent release of the inflammatory mediators. As for the role of soluble LIGHT, however, no remarkable differences of plasma LIGHT levels were found between stable plaque and unstable plaque subgroup in the stroke patients, despite a trend towards a decrease in the plasma protein levels following treatment of aspirin, one of the GP IIb/IIIa antagonists. Our data consequently support the notion that soluble LIGHT protein, once released from platelets and monocytes/macrophages, may predominantly functions as one pro-inflammatory cytokine in the inflammatory process of acute atherosclerotic stroke. These data, however, should be interpreted with high precaution due to the limited number of patients included in our serial study. On the other hand, the immunologic derangement of soluble LIGHT may alternatively be a general phenomenon in atherogenetic disorders, as its elevated levels have been also shown in some cardiovascular diseases [13] [14].

This study shows a remarkable dysregulation of LIGHT expression in peripheral blood of patients with acute ischemic atherosclerotic stroke, reflecting a persistent chronic inflammatory response that may have been induced during early stages of the disease. Our results strongly suggest distinctive roles of the form-associated LIGHT in acute ischemic atherosclerotic stroke: platelet-associated LIGHT may contribute to formation and development of carotid atherosclerotic plaque, probably involving plaque destabilization, while soluble LIGHT may predominantly participate in the acute inflammatory process of stroke. Further studies on the influence of different etiologies of ischemic stroke and other atherothrombotic diseases on the effects of the form-associated LIGHT are needed to gain more insight into the pathophysiological significance of this TNFSF member in human stroke.

\section{Acknowledgements}

We thank Dr Yan Chang for her technical assistance with flow cytometry. We also thank Dr Gabriel Linares for his careful discussion during the work. This work was supported by grants from Peking University People's Hospital (RDB2009-31), the National Natural Science fund (NSF 81171123), the Swedish Research Council (grant no.11220) and the Swedish Association of Neurologically Disabled.

\section{References}

[1] Elkind, M.S. (2006) Inflammation, Atherosclerosis and Stroke. Neurologist, 12, 140-148. 
http://dx.doi.org/10.1097/01.nrl.0000215789.70804.b0

[2] Weber, C. and Noels, H. (2011) Atherosclerosis: Current Pathogenesis and Therapeutic Options. Nature Medicine, 17, 1410-1422. http://dx.doi.org/10.1038/nm.2538

[3] Libby, P. (2002) Inflammation in Atherosclerosis. Nature, 420, 868-874. http://dx.doi.org/10.1038/nature01323

[4] Buch, M.H., Prendergast, B.D. and Storey, R.F. (2010) Antiplatelet Therapy and Vascular Disease, an Update. Therapeutic Advances in Cardiovascular Disease, 4, 249-275. http://dx.doi.org/10.1177/1753944710375780

[5] Gerdes, N. and Zirlik, A. (2011) Co-Stimulatory Molecules in and beyond Co-Stimulation-Tipping the Balance in Atherosclerosis? Thrombosis and Haemostasis, 106, 804-813. http://dx.doi.org/10.1160/TH11-09-0605

[6] Hassan, G.S., Merhi, Y. and Mourad, W. (2012) CD40 Ligand: A Neo-Inflammatory Molecule in Vascular Diseases. Immunobiology, 217, 521-532. http://dx.doi.org/10.1016/j.imbio.2011.03.010

[7] Young, J.L., Libby, P. and Schonbeck, U. (2002) Cytokines in the Pathogenesis of Atherosclerosis. Thrombosis and Haemostasis, 88, 554-567.

[8] McKellar, G.E., McCarey, D.W., Sattar, N. and McInnes, I.B. (2009) Role for TNF in Atherosclerosis? Lessons from Autoimmune Disease. Nature Reviews Cardiology, 6, 410-417. http://dx.doi.org/10.1038/nrcardio.2009.57

[9] Lee, W.H., Kim, S.H., Lee, Y., Lee, B.B., Kwon, B. and Park, J.E. (2001) Tumor Necrosis Factor Receptor Superfamily 14 Is Involved in Atherogenesis by Inducing Proinflammatory Cytokines and Matrix Metalloproteinases. Arteriosclerosis, Thrombosis, and Vascular Biology, 21, 2004-2010. http://dx.doi.org/10.1161/hq1201.098945

[10] Otterdal, K., Smith, C., Oie, E., Pedersen, T.M., Yndestad, A., Stang, E., Endresen, K., Solum, N.O., Aukrust, P. and Damås, J.K. (2006) Platelet-Derived LIGHT Induces Inflammatory Responses in Endothelial Cells and Monocytes. Blood, 108, 928-935. http://dx.doi.org/10.1182/blood-2005-09-010629

[11] Kim, W.J., Kang, Y.J., Suk, K., Park, J.E., Kwon, B.S. and Lee, W.H. (2008) Comparative Analysis of the Expression Patterns of Various TNFSF/TNFRSF in Atherosclerotic Plaques. Immunological Investigations, 37, 359-373. http://dx.doi.org/10.1080/08820130802123139

[12] Chang, Y.H., Hsieh, S.L., Chao, Y., Chou, Y.C. and Lin, W.W. (2005) Proinflammatory Effects of LIGHT through HVEM and LT $\beta R$ Interactions in Cultured Human Umbilical Vein Endothelial Cells. Journal of Biomedical Science, 12, 363-375. http://dx.doi.org/10.1007/s11373-005-1360-5

[13] Scholz, H., Sandberg, W., Damas, J.K., Smith, C., Andreassen, A.K., Gullestad, L., Frøland, S.S., Yndestad, A., Aukrust, P. and Halvorsen, B. (2005) Enhanced Plasma Levels of LIGHT in Unstable Angina: Possible Pathogenic Role in Foam Cell Formation and Thrombosis. Circulation, 112, 2121-2129. http://dx.doi.org/10.1161/CIRCULATIONAHA.105.544676

[14] Celik, S., Langer, H., Stellos, K., May, A.E., Shankar, V., Kurz, K., Katus, H.A., Gawaz, M.P. and Dengler, T.J. (2007) Platelet-Associated LIGHT (TNFSF14) Mediates Adhesion of Platelets to Human Vascular Endothelium. Thrombosis and Haemostasis, 98, 798-805.

[15] Liu, G.Z., Fang, L.B., Hjelmstrom, P. and Gao, X.G. (2008) Enhanced Plasma Levels of LIGHT in Patients with Acute Atherothrombotic Stroke. Acta Neurologica Scandinavica, 118, 256-259. http://dx.doi.org/10.1111/j.1600-0404.2008.01013.x

[16] Adams Jr., H.P., Bendixen, B.H., Kappelle, L.J., Biller, J., Love, B.B., Gordon, D.L. and Marsh, E.E. (1993) Classification of Subtype of Acute Ischemic Stroke. Definitions for Use in a Multicenter Clinical Trial. TOAST. Trial of Org 10172 in Acute Stroke Treatment. Stroke, 24, 35-41. http://dx.doi.org/10.1161/01.STR.24.1.35

[17] Spence, J.D., Eliasziw, M., DiCicco, M., Hackam, D.G., Galil, R. and Lohmann, T. (2002) Carotid Plaque Area: A Tool for Targeting and Evaluating Vascular Preventive Therapy. Stroke, 33, 2916-2922. http://dx.doi.org/10.1161/01.STR.0000042207.16156.B9

[18] Spence, J.D. (2006) Technology Insight: Ultrasound Measurement of Carotid Plaque—Patient Management, Genetic Research, and Therapy Evaluation. Nature Clinical Practice Neurology, 2, 611-619. http://dx.doi.org/10.1038/ncpneuro0324

[19] Cohavy, O., Zhou, J., Ware, C.F. and Targan, S.R. (2005) LIGHT Is Constitutively Expressed on T and NK Cells in the Human Gut and Can Be Induced by CD2-Mediated Signaling. The Journal of Immunology, 174, 646-653. http://dx.doi.org/10.4049/jimmunol.174.2.646

[20] Schneider, K., Potter, K.G. and Ware, C.F. (2004) Lymphotoxin and LIGHT Signaling Pathways and Target Genes. Immunological Reviews, 202, 49-66. http://dx.doi.org/10.1111/j.0105-2896.2004.00206.X

[21] del Rio, M.L., Lucas, C.L., Buhler, L., Rayat, G. and Rodriguez-Barbosa, J.I. (2010) HVEM/LIGHT/BTLA/CD160 Cosignaling Pathways as Targets for Immune Regulation. Journal of Leukocyte Biology, 87, 223-235. http://dx.doi.org/10.1189/jlb.0809590

[22] Celik, S., Shankar, V., Richter, A., Hippe, H.J., Akhavanpoor M., Bea, F., Erbel, C., Urban, S., Blank, N., Wambs- 
ganss, N., Katus, H.A. and Dengler, T.J. (2009) Proinflammatory and Prothrombotic Effects on Human Vascular Endothelial Cells of Immune-Cell-Derived LIGHT. European Journal of Medical Research, 14, 147-156.

http://dx.doi.org/10.1186/2047-783X-14-4-147

[23] Garlichs, C.D., Kozina, S., Fateh-Moghadam, S., Handschu, R., Tomandl, B., Stumpf, C., Eskafi, S., Raaz, D., Schmeisser, A., Yilmaz, A., Ludwig, J., Neundörfer, B. and Daniel, W.G. (2003) Upregulation of CD40-CD40 Ligand (CD154) in Patients with Acute Cerebral Ischemia. Stroke, 34, 1412-1418. http://dx.doi.org/10.1161/01.STR.0000074032.64049.47

[24] Garrido, V.T., Proença-Ferreira, R., Dominical, V.M., Traina, F., Bezerra, M.A., de Mello, M.R., Colella, M.P, Araújo, A.S., Saad, S.T., Costa, F.F. and Conran, N. (2012) Elevated Plasma Levels and Platelet-Associated Expression of the Pro-Thrombotic and Pro-Inflammatory Protein, TNFSF14 (LIGHT), in Sickle Cell Disease. British Journal of Haematology, 158, 788-797. http://dx.doi.org/10.1111/j.1365-2141.2012.09218.x 
Scientific Research Publishing (SCIRP) is one of the largest Open Access journal publishers. It is currently publishing more than 200 open access, online, peer-reviewed journals covering a wide range of academic disciplines. SCIRP serves the worldwide academic communities and contributes to the progress and application of science with its publication.

Other selected journals from SCIRP are listed as below. Submit your manuscript to us via either submit@scirp.org or Online Submission Portal.
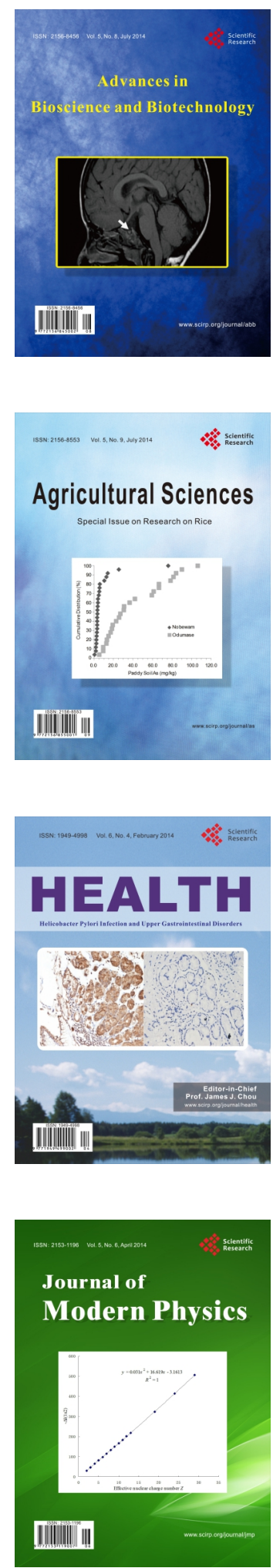
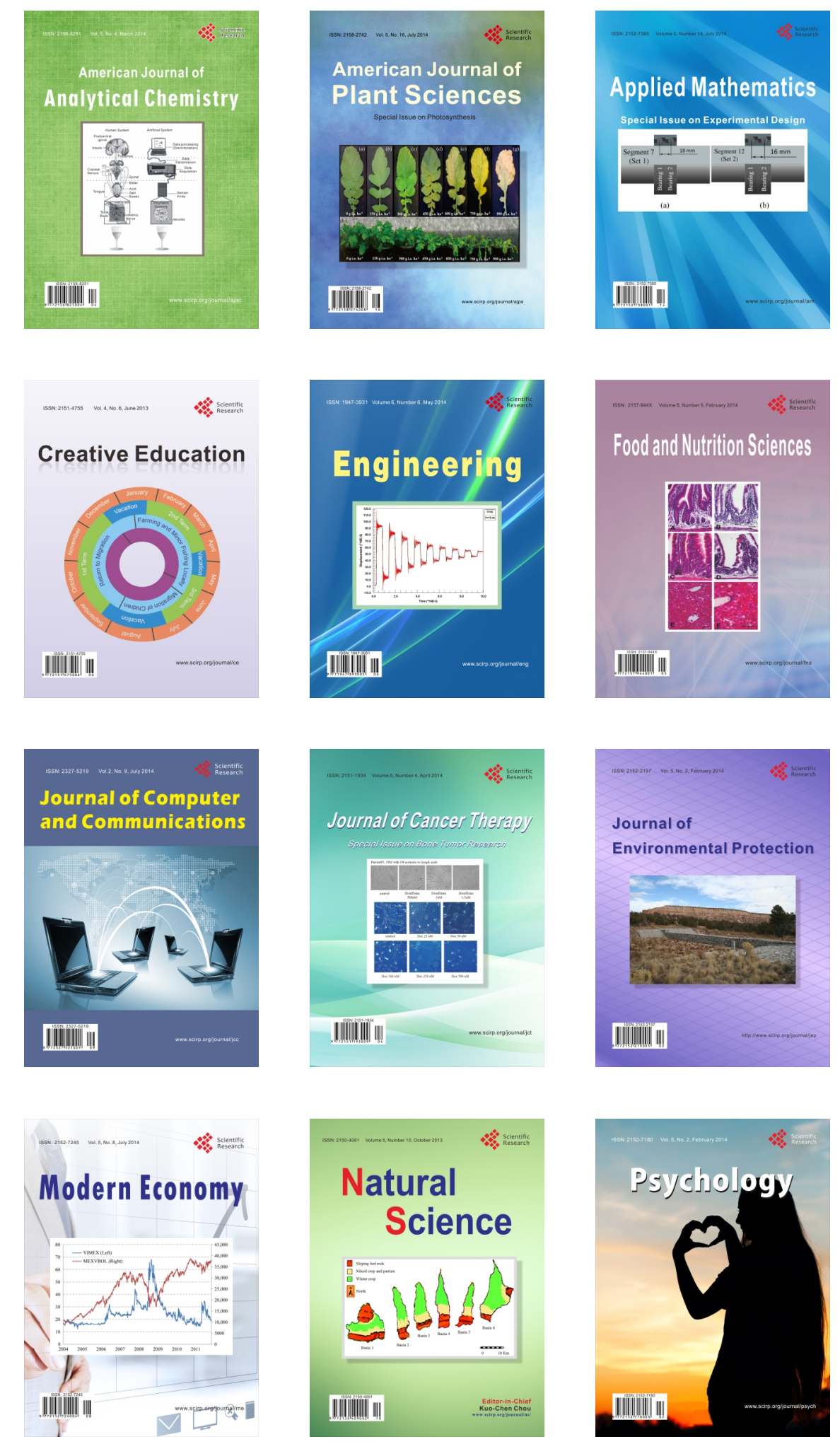